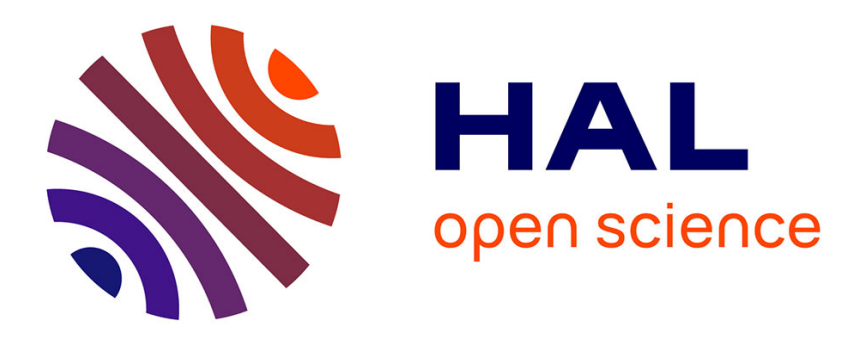

\title{
Le vol en interne: les vols commis par les salariés sur leur lieu de travail
}

François Bonnet

\section{To cite this version:}

François Bonnet. Le vol en interne: les vols commis par les salariés sur leur lieu de travail. Sociologie du Travail, 2007, 49 (4), pp.544-556. halshs-00879307

\section{HAL Id: halshs-00879307 https://shs.hal.science/halshs-00879307}

Submitted on 4 Nov 2013

HAL is a multi-disciplinary open access archive for the deposit and dissemination of scientific research documents, whether they are published or not. The documents may come from teaching and research institutions in France or abroad, or from public or private research centers.
L'archive ouverte pluridisciplinaire HAL, est destinée au dépôt et à la diffusion de documents scientifiques de niveau recherche, publiés ou non, émanant des établissements d'enseignement et de recherche français ou étrangers, des laboratoires publics ou privés. 


\section{Le vol en interne : les vols commis par les salariés sur leur lieu de travail}

François Bonnet

frabonnet@gmail.com

\section{Résumé}

Ce travail fait le point sur la littérature en sciences sociales sur le vol en interne (les vols commis par les salariés sur leur lieu de travail). Les enquêtes ethnographiques et par auto-révélation montrent qu'il s'agit d'une déviance prégnante dans le monde du travail. Les sociologues et les anthropologues interprètent principalement le vol en interne comme un moyen politique de résistance contre l'injustice de l'exploitation au travail. Symétriquement, les économistes et la littérature du management insistent sur la dimension proprement délinquante du vol et recherchent les moyens pour les patrons de se prémunir contre les salariés indélicats. Au-delà de cette opposition, le vol en interne apparaît comme un cas pertinent pour étudier la complexité du rapport salarial.

Mots-clés : vol en interne, fraude interne, déviance au travail, conflit au travail, microéconomie 


\section{Le vol en interne : les vols commis par les salariés sur leur lieu de travail}

\section{Le vol en interne : une délinquance sur le lieu de travail}

Sur le lieu de travail, les salariés peuvent commettre quantité de comportements répréhensibles. Robinson et Bennett (1995: 565) ont dressé une typologie des comportements déviants au travail, en les classant selon leur gravité, et selon qu'ils concernent les personnes ou l'organisation. Le vol en interne - les vols commis par les salariés sur leur lieu de travail - ne concerne qu'une partie de toutes les déviances potentielles.

Déviances contre l'organisation

\begin{tabular}{|c|c|c|c|}
\hline \multirow{3}{*}{$\begin{array}{l}\text { Déviances } \\
\text { mineures }\end{array}$} & $\begin{array}{c}\text { Déviances relatives à la } \\
\text { production }\end{array}$ & Déviances relatives à la propriété & \multirow{4}{*}{$\begin{array}{l}\text { Déviances } \\
\text { sérieuses }\end{array}$} \\
\hline & $\begin{array}{c}\text { Partir en avance } \\
\text { Prendre des pauses excessives } \\
\text { Travailler en dessous de sa } \\
\text { productivité } \\
\text { Gâcher des ressources }\end{array}$ & $\begin{array}{c}\text { Sabotage } \\
\text { Accepter la corruption } \\
\text { Mentir à propos des heures } \\
\text { travaillées } \\
\text { Voler l'entreprise }\end{array}$ & \\
\hline & Déviances politiques & Agressions personnelles & \\
\hline & $\begin{array}{l}\text { Pratiquer le favoritisme } \\
\text { Commérer } \\
\text { Dénigrer les collègues } \\
\text { Compétition stérile }\end{array}$ & $\begin{array}{c}\text { Harcèlement sexuel } \\
\text { Agression verbale } \\
\text { Voler les collègues } \\
\text { Mettre en danger les collègues }\end{array}$ & \\
\hline
\end{tabular}

Déviances contre les personnes

Le « vol » est un terme très général, qui englobe le vol d'argent, de biens, de services (téléphone, Internet) et d'informations (Lipman et McGraw 1988 : 5354). Cette définition n'englobe pas le vol de temps, comme le fait de prendre des pauses abusives, de bavarder avec ses collègues ou de faire semblant de travailler devant son ordinateur ${ }^{1}$. Le terme "vol en interne » ressort du vocabulaire patronal et n'a de sens que par l'opposition avec les vols « en externe », c'est-à-dire les vols commis par les clients. La criminologie américaine dispose d'une terminologie plus précise. Elle distingue d'abord plusieurs grandes catégories de criminalités : le street crime (les agressions et les vols), le domestic crime (les violences conjugales) et le white-collar crime (les crimes commis autour du lieu de travail). Au sein de la catégorie générique white-collar crime, il faut distinguer le corporate crime - ce que nous appelons la délinquance en col blanc, c'est-àdire les crimes commis par les patrons et les cadres à l'encontre des salariés ou des clients, de l'occupational crime, qui concerne spécifiquement la criminalité des salariés contre l'entreprise. Parmi les délinquances des salariés, la criminologie américaine parle d'employee theft pour désigner le vol en interne, ou d'embezzlement, qui induit l'idée que les salariés abusent de la confiance que

\footnotetext{
${ }^{1}$ Le vol de temps est considéré par les entreprises comme un grave problème ; une part importante de la productivité des enseignes Wal-Mart aux Etats-Unis proviendrait de la capacité de l'encadrement à empêcher les salariés de «gaspiller » du temps de travail (Ehrenreich, 2002).
} 
l'encadrement place en eux (Beirne et Messerschmidt 2006 : 195 et sq. ; Barkan $2001: 330$ et sq; Siegel $2003: 393$ ).

Ce travail entend faire le point sur la littérature existante sur le vol en interne. Cette littérature est presque totalement anglaise ou américaine; à ma connaissance, la sociologie française s'est intéressée plus spécifiquement à la «perruque » (Joseph 1980, Anteby 2003) qu'aux pratiques frauduleuses d'appropriation des biens de l'entreprise. En fait, le seul article français sur la question (Bozon et Lemel 1989) comprend cinq pages sur le vol (pp. 113-117) sans jamais utiliser le mot, préférant parler avec pudeur de «récupération », «emprunts provisoires ou définitifs », voire «appropriation pure et simple ». Au regard du contexte social et politique français, il n'est pas absurde d'imaginer que les sociologues du travail français se sont trouvés dans l'embarras devant une délinquance doublement problématique: problématique pour la revendication collective (un salarié-voleur est moins légitime qu'un salarié vertueux), et problématique pour la sociologie : si des salariés intégrés (et à fortiori des cadres et des patrons) volent, comment faire reposer la cause du crime sur le chômage et la pauvreté ? De fait, la littérature sur le vol en interne est structurée par le caractère problématique de cette délinquance, avec une opposition nette entre les approches sociologiques et les approches des sciences du management.

Pour organiser cette revue de littérature, on va présenter les différentes méthodologies utilisées pour enquêter le phénomène et les types de données produits (2). En tant que champ de recherche, l'intérêt principal du vol en interne est de montrer le clivage presque caricatural entre d'une part les comptes-rendus de la sociologie et de l'anthropologie, qui tendent à analyser le vol en interne comme une résistance à l'oppression patronale (3), et les comptes-rendus de l'économie et du management d'autre part, qui insistent avec la même vigueur sur les dommages causés par le vol et sur la malignité des employés (4). Des pistes d'études laissent cependant présager de la fécondité du cas du vol en interne pour comprendre la complexité du rapport salarial (5).

\section{Enquêter le vol en interne : les différentes méthodologies}

Les recherches sur le vol en interne s'appuient sur plusieurs types de données : les trois principales méthodologies sont les ethnographies de lieux de travail, les enquêtes par auto-révélation auprès d'un échantillon de salariés et les enquêtes de victimation.

\section{1 Les ethnographies du lieu de travail}

Les ethnographies du lieu de travail ont un intérêt multiple. Le premier est d'opérer des distinctions pertinentes des différents types de vols en fonction des contextes pratiques du travail salarié. Par exemple, Ditton (1977a et 1977b) a travaillé pendant plusieurs années dans une boulangerie industrielle. Il distingue le chapardage du vol proprement dit. Voler un peu de nourriture ou des chutes de papier-toilette de temps à autre pour son propre usage, comme «salaire invisible », n'est pas du même ressort qu'organiser le recel et la revente de marchandises volées à grande échelle, pour constituer un revenu. Les salaires invisibles de Ditton sont les moyens auxquels les employés ont recours pour améliorer leurs conditions de travail et leur revenu (sans passer par la négociation collective), et se divisent en trois catégories. Les «à-côtés» (perks) sont les 
avantages accessoires liés à chaque métier, par exemple les canettes de soda gratuites des vendeuses de denrées alimentaires en kiosque. Le chapardage (pilferage) recouvre toutes les formes de vol en interne, par exemple les fournitures de bureaux. Les combines (fiddle) sont les petites escroqueries quotidiennes que les salariés pratiquent aux dépends des clients, comme mal rendre la monnaie et garder la différence.

L'anthropologue Mars (1982) préfère établir une typologie des métiers auxquels sont associés des formes particulières de vol.

\begin{tabular}{cc|c}
\multicolumn{2}{c}{ Travail isolé } \\
\multirow{2}{*}{$\begin{array}{c}\text { Surveillance étroite et } \\
\text { salariés isolés sur le lieu de } \\
\text { travail : caissières, ouvriers à } \\
\text { la chaîne }\end{array}$} & $\begin{array}{c}\text { Forte autonomie et salariés } \\
\text { travaillant seuls : cadres, } \\
\text { journalistes }\end{array}$ \\
\cline { 2 - 3 }+ & $\begin{array}{c}\text { Surveillance étroite et travail } \\
\text { en groupe : serveurs, } \\
\text { personnel hôtelier }\end{array}$ & $\begin{array}{c}\text { Relative autonomie et travail } \\
\text { en groupe }: \text { dockers, } \\
\text { bagagistes }\end{array}$ \\
Travail en groupe
\end{tabular}

Les salariés isolés et peu autonomes dans leur travail, comme les caissières dans les supermarchés ou certains ouvriers, ne peuvent organiser des combines élaborées. Par exemple, les caissières font passer leurs proches à la caisse sans les faire payer, en faisant semblant de faire bipper le code barre. Ces salariés sont par ailleurs les spécialistes de l'absentéisme (Mars 1982 : 66-88). Les employés qui travaillent en groupe et sont peu autonomes, comme les serveurs dans les cafés et le personnel hôtelier, comptent sur leurs collègues pour fermer les yeux sur leurs larcins personnels. Une combine typique est d'allonger les cafés demandés par les clients dans les hôtels avec de l'eau. Un serveur qui doit apporter quatre cafés à une table en commande deux, les coupe pour en faire quatre, et encaisse l'argent des deux cafés supplémentaires (Mars 1982 : 108135). Ceux qui travaillent en groupe et parviennent à développer un peu d'autonomie organisent généralement des combines de groupes, comme les dockers. Ils contraignent d'abord l'encadrement à recruter des proches. Ceux-ci feront grève de façon solidaire si l'un d'eux est accusé de vol en interne. Ils se partagent les tâches pour voler plus et mieux : les uns distrayant l'encadrement tandis que les autres volent les marchandises qu'ils doivent décharger. Souvent, les travailleurs qui volent en groupe développent des normes qui interdisent les vols individuels (Mars 1982 : 89-107). Selon Dickens et al. (1989 : 302), 80 \% des pertes inexpliquées dans la marine marchande, sur les docks et dans les aéroports seraient imputables au vol en interne. Les salariés qui travaillent seuls et sont indépendants (les cadres) exploitent leur autonomie aux dépens de l'organisation. Par exemple, les journalistes facturent des fausses notes de frais quand ils vont au restaurant avec leurs amis. Les cadres profitent de leurs voyages professionnels pour faire du tourisme (Mars 1982 : 40-65).

Les ethnographies de lieux de travail documentent des pratiques qu'on ne soupçonnerait pas, et montrent comment les salariés sont ingénieux et imaginatifs pour voler en dépit des contraintes. Elles permettent de voir comment le vol en interne est une délinquance routinière ; comme dit Mars (1982 : 20), « cependant, 
une proportion considérable [de données] fut collectée par la simple méthode de garder les yeux et les oreilles ouverts ».

\subsection{Les enquêtes par auto-révélation}

Dans les enquêtes par auto-révélation, on demande aux enquêtés euxmêmes de reporter leurs crimes et délits (au contraire des enquêtes de victimation, où on demande aux enquêtés de reporter les crimes et délits subis). Dans le cas du vol en interne, il s'agit de dénombrer les salariés-voleurs et de trouver les corrélations pertinentes entre vols et variables indépendantes.

Des sociologues et des psychologues ont mené des enquêtes par autorévélation sur le vol en interne. Hollinger et Clark (1983: 401) ont conduit une enquête aux Etats-Unis $(\mathrm{N}=9175)$ qui montre que de 28,4 à $35,1 \%$ des employés (selon les secteurs d'activité) volent ou fraudent dans leur intérêt personnel au moins une à trois fois par an. Le vol ou la fraude sur une base quotidienne est marginal et n'est pas avoué par plus de $0,2 \%$ des employés, quels que soient les secteurs. En France, Bozon et Lemel (1989 : 116) rapportent que 35\% des salariés investigués $(\mathrm{N}=446)$ ramènent chez eux des rebuts, des matériaux ou des fournitures de l'entreprise, la proportion variant de $26 \%$ pour les ouvrières à $44 \%$ pour les ouvriers. En Chine, Kwok et al. (2005 : 463) ont trouvé des proportions similaires $-1 / 3$ d'employés voleurs auto-déclarés, avec un échantillon plus réduit $(\mathrm{N}=155)$. D'autres enquêtes par auto-révélation ont été menées, avec des objectifs différents. Tucker (1993) a interrogé 250 étudiants sur leurs expériences de travail pour savoir comment ils exprimaient leur insatisfaction au travail, le vol en interne étant une des modalités de « résistance ».

Les enquêtes par auto-révélation induisent un biais de conformité important : les enquêtés peuvent toujours craindre que leurs réponses ne sont pas vraiment anonymes et que tout ce qu'ils pourront avouer sera retenu contre eux. Elles sont cependant utiles pour estimer l'ampleur du vol en interne.

\subsection{Les enquêtes de victimation}

Les enquêtes de victimation sont des questionnaires adressés aux victimes potentielles afin de compléter les dispositifs habituels de recension de la criminalité (que sont les statistiques de la police et les enquêtes par autorévélation). Dans le cadre du vol en interne, les victimes directes sont les patrons. Blount (2003), Payne et Gainey (2004 : 70) ont montré que le vol en interne a des conséquences que ces auteurs jugent «dévastatrices» pour les patrons des magasins les plus touchés. Ces patrons souffraient de façon disproportionnée de stress, de fatigue, et de perte de confiance envers la société, les institutions et la justice.

Pour les patrons, le vol en interne fait partie de la «démarque inconnue », c'est-à-dire l'ensemble des pertes inexpliquées : les vols commis par les clients, les vols commis par les employés, et les erreurs administratives. Selon la sagesse patronale, "la démarque c'est un tiers / un tiers / un tiers »: il y a autant de vol en interne que de vol commis par la clientèle (Shapland 1995 : 323). Ocqueteau et Pottier (1995 : 120-123) estiment à seulement $20 \%$ la part des vols commis par les clients dans la démarque inconnue dans le supermarché qu'ils ont enquêté. En France, 42,1 \% des responsables de grandes surfaces interrogés dans 
une enquête nationale de 1994 estimaient que le vol en interne est une réalité « plus préoccupante » que par le passé (Ocqueteau et Pottier, 1995 : 64).

Les enquêtes de victimation permettent d'intégrer le point de vue de l'encadrement dans la perception du vol en interne. Le fait que les patrons se considèrent comme des victimes n'est pas absurde au regard des sommes en jeu. Les estimations du coût du vol en interne sont très variables, mais des économistes estiment que les transferts de richesse de l'entreprise vers le salarié indélicat seraient chaque année aux Etats-Unis compris entre 15 et 56 milliards de dollars, contre 4 milliards de dollars pour la criminalité ordinaire (street crime) (Dickens et al. 1989). Dans le monde de la banque, il a été estimé que les employés avaient volé 382 millions de dollars en 1982, soit neuf fois plus que le montant volé par les braqueurs (Lipman et McGraw 1988 : 52).

\section{La sociologie du vol en interne et l'idée de résistance}

Le fait marquant de la littérature sur le vol en interne, c'est que la quasitotalité des travaux de sociologie ou d'anthropologie montrent un parti pris affirmé en faveur des employés qui volent. Les théories sociologiques ou anthropologiques du vol en interne minimisent la dimension criminelle des vols ou fournissent des justifications aux salariés en étudiant le vol en interne comme une manifestation de la vitalité de la contestation dans l'entreprise.

Dans la perspective de la minimisation de la dimension criminelle, les théories de la sous-culture délinquante (Sutherland 1924 et Sellin 1938) ont été ressuscitées par Horning (1970) et Sieh (1987). Horning montre que les employés entretiennent des normes qui tolèrent ou encouragent le vol. Ces normes sont fondées sur une forme de solidarité des travailleurs face au patron. Sieh a observé que lorsque les syndicats sont perçus comme inefficaces et corrompus, les employés se constituent en groupes dont la solidarité est fondée sur des normes favorables aux comportements déviants sur le lieu de travail. La théorie de la frustration (Merton 1938) qui, en criminologie, répond aux théories de la sousculture, a été abondamment utilisée pour expliquer le vol en interne (Spector 1997), soit que les salariés n'aiment pas leur travail dans l'absolu (Mangione et Quinn 1975), soit qu'ils s'estiment moins bien payés que leurs collègues pour un travail équivalent (Sieh 1993).

Mais pour la plupart, les sociologues ont conceptualisé le vol en interne comme un acte politique de résistance. Comme le remarquent Tucker (1989; 1993 ; 1999), Ackroyd et Thompson (1999 : 31-52), Morrill et al., (2003 : 394397), Hodson (2004) et Roscigno et Hodson (2004 : 16), les formes de résistance des salariés ne se limitent pas à l'expression syndicale et à la grève. Le sabotage et le vol, «plutôt que de simples actes de délinquance, reflètent souvent des griefs plus larges à propos de l'organisation et des conditions du travail » (Roscigno et Hodson, 2004 : 14).

Pour donner du corps à l'hypothèse selon laquelle le vol en interne exprime «plus » que le simple appât du gain, un psychologue, Greenberg (1990 : 562) a voulu montrer que le vol en interne était corrélé à l'injustice perçue au travail. Il a bénéficié d'un protocole empirique idéal : trois usines d'une même entreprise, peuplées d'ouvriers aux caractéristiques démographiques semblables, 
situées dans des territoires comparables, dont deux doivent imposer une baisse de salaire de $15 \%$ en raison de la perte d'un contrat commercial. Dans l'une des deux usines où le salaire est réduit, le directeur vient en personne devant les ouvriers et annonce la nouvelle avec beaucoup d'humanité, en insistant sur le fait que l'encadrement subira aussi cette baisse de salaire, et enjoint les ouvriers de venir le rencontrer personnellement pour parler des problèmes que cette mesure pourrait causer. Dans l'autre usine, le sous-directeur annonce la mesure de façon sèche et conclut en disant qu'il doit partir prendre un avion (Greenberg 1990: 563). Greenberg mesure l'évolution des pertes inexpliquées dans l'usine sur la durée de la mise en œuvre de la réduction de salaire (quelques mois) en contrôlant les résultats avec ceux de l'usine où aucune réduction de salaire n'a été décidée. Le taux de perte (le vol en interne) croît fortement dans l'usine où l'explication de la réduction de salaire a été sèche, et reste stable dans les deux autres usines (Greenberg 1990 : 566), montrant que le vol en interne peut être interprété comme une forme d'expression d'insatisfaction des salariés.

Des sociologues plus radicaux ont interprété le vol en interne comme un moyen à la disposition des salariés pour contrôler l'encadrement. Tucker (1993) se réfère au cadre théorique d'étude du contrôle social mis en place par Donald Black au début des années quatre-vingt (Black, dir. 1984a et 1984b). Black inscrit le contrôle social dans une perspective relationnelle dans laquelle le crime luimême peut être conçu comme une forme de contrôle social. Il estime que «beaucoup de crimes appartiennent à la même famille que le commérage, la dérision, la vengeance, le châtiment, et la loi elle-même ». Le caractère «criminel » du crime ne dérive que de ce que la loi le punit; par exemple, la moitié des homicides à Houston en 1969 relèveraient de la provocation ou de la légitime défense (Black 1983 : 36, 42). Baumgartner a systématisé cette intuition en théorisant le «contrôle social d'en bas » (social control from below). Le contrôle social a trop été vu selon elle comme le contrôle des faibles et des exploités par les puissants et les nantis : «là où il y a des inégalités sociales, et ailleurs, l'ordre normatif est défini et maintenu par un processus interactif, et les supérieurs sont rarement complètement libres de pression morale venue d'en bas » (Baumgartner 1984 : 304). Le social control from below comprend bien sûr les émeutes, mais aussi les représailles anonymes. Parmi les différentes formes de contrôle social bottom-up, le travail de Tucker (1993) se concentre sur «les formes quotidiennes de résistance des employés », telles que les ragots, la confrontation directe organisée ou non, le sabotage, ou l'absentéisme, et parmi elles, le vol en interne. Tucker considère le vol en interne comme une forme de contrôle social, un moyen qu'ont les salariés de manifester leur mécontentement et de punir leur encadrement (Tucker 1989).

Dans la même veine, Morrill et al. (2003 : 392) insistent sur l'importance des «conflits politiques cachés » dans les organisations pour les groupes dominés (les salariés), car ils permettent d' «exprimer leurs griefs » et de «déployer des formes tacites ou explicites de solidarité ». Bies et al. (1997 : 32) estiment que la peur de la vengeance (sous forme de vol et de sabotage) est le moyen efficace de la bonne conduite de l'encadrement: vu sous cet angle, le vol en interne est une déviance «constructive » et «prosociale ». Pour l'anthropologue Mars (1982), il 
n'est pas exagéré de voir le vol en interne comme une «déviance réciproque »: non un crime, mais une compensation due pour l'exploitation ${ }^{1}$.

\section{Le vol en interne pensé par l'économie et le management}

L'interprétation du vol en interne en termes de résistance est confortable pour les sociologues qui veulent prendre la défense des salariés, mais elle peut être accusée de naïveté. Postuler que tous les vols en interne sont des actes de résistance est irréaliste. Les salariés, qui ne sont pas des «idiots culturels", peuvent instrumentaliser le prétexte de la résistance et rationalisent leur inconduite (Bernardin et Cooke 1993 : 1098 ; Kwok et al. 2005 : 457). Les sociologues de la déviance ont bien identifié la tendance des délinquants à rationaliser leurs conduites par des «techniques de neutralisation » (Sykes et Matza 1957) : minimiser le tort causé, reporter la responsabilité du tort sur la victime, nier la légitimité des condamnateurs ou se référer à des loyautés supérieures. Katz (1988) a en outre décrit les « séductions du crime », c'est-à-dire le plaisir, l'excitation et les satisfactions psychologiques que l'on peut ressentir à commettre un acte délinquant - sans que cela ait le moindre rapport avec la contestation politique.

Dans cette perspective, et symétriquement par rapport au parti pris de la sociologie, les revues d'économie, de psychologie appliquée, de management et de droit conçoivent le vol en interne comme un crime contre l'entreprise et contre sa rentabilité. En conséquence, l'étiologie du vol en interne se focalise sur la responsabilité individuelle des contrevenants. Le reste de la recherche porte sur les méthodes efficaces pour réduire le vol dans son entreprise ou sur les conséquences non-économiques du vol en interne pour ses victimes, les patrons. La problématique qui organise les travaux animés par cette conception est typiquement positiviste : déterminer les causes pour réduire le crime.

Pour les économistes, le vol en interne est une pure nuisance : il nuit à la maximisation du profit par l'entreprise, à la fois par le préjudice qu'il entraîne, mais aussi par les coûts de surveillance qu'il induit. Du point de vue théorique, le vol en interne est un cas classique de problème du principal-agent. Le problème du principal-agent a été formalisé en microéconomie (pour une analyse sociologique : Shapiro 2005). Il sert à caractériser les propriétés d'une situation d'information asymétrique entre deux acteurs qui rentrent dans une relation d'échange. L'exemple fictif est celui du principal (d'un collège) qui donne un travail à accomplir à son agent (d'entretien). Réaliser ce travail est utile au principal mais diminue la satisfaction de l'agent. Le principal ne peut surveiller tout ce que fait son agent, parce que cela est trop coûteux ou impossible. Il y a donc un problème de base dans la relation, puisque le principal ne peut jamais être sûr que l'agent fait bien son travail, tout en devant continuer à le payer. Pour Dickens et al. (1989), la solution pareto-optimale au problème du vol en interne est soit (1) de surveiller au minimum mais d'infliger des peines d'une sévérité extrême, afin de dissuader à faible coût, soit (2) de payer des salaires d'efficience aux salariés, qui seraient incités à ne pas risquer de perdre leur travail.

${ }^{1}$ Sur une base purement spéculative, Ackroyd et Thompson (1999: 144-165) et Tucker (1999: 12-13) prédisent le déclin de la déviance sur le lieu de travail. Ils estiment que la qualité des relations entre salariat et encadrement s'inscrit dans une tendance historique à l'amélioration. De là, le volume de déviance sur le lieu de travail est voué à décliner. 
Dickens et al. (1989) observent que dans le monde réel, les entreprises dépensent des sommes considérables pour la surveillance ; comment en expliquer les raisons ? Le problème de la sévérité excessive est qu'elle peut être limitée par le droit du travail ; qu'elle peut heurter le sentiment tacite de justice des salariés, et donc leur motivation ; surtout, la possibilité d' «erreurs judiciaires » de la part de l'entreprise risque d'inciter les salariés à passer plus de temps à se prémunir contre une possible erreur qu'à travailler pour l'entreprise. Le problème des salaires d'efficience découle du principe même de la structure de relation principal-agent: l'employeur ne peut se permettre de payer des salaires d'efficience à tous les salariés sans savoir qui est vertueux et qui ne l'est pas, sous peine d'inciter tout le monde à voler - cas classique de moral hazard. En somme, Dickens et al. (1989) montrent que les entreprises sont condamnées à supporter les coûts de la surveillance : par exemple, de nombreuses commerces de détail tolèrent que les salariés «se servent », ou bénéficient de prix plus avantageux. Mais cette tactique n'est opérante que si la diminution induite du coût de la surveillance est supérieure au coût de la tolérance; ce qui, en toute rigueur microéconomique, suppose le problème résolu a priori.

Astor (1972) produit un argument proche de l'économie classique en affirmant que le vol en interne dépend fondamentalement des opportunités disponibles présentes sous les yeux des employés. Kwok et al. (2005: 471), qui étudient des entreprises chinoises, entendent montrer que la propension des employés au vol dépend du contrôle formel dont ils font l'objet: plus les employés sont surveillés, plus le vol devient risqué, et moins les employés volent. Pour Dickens et al., Astor et Kwok et al., l'employé est un acteur rationnel qui décide de voler ou non en fonction des opportunités disponibles et de la probabilité d'être découvert, et la définition de ce qui est considéré par une société donnée comme un crime ne pose aucun problème théorique. Cette conception renvoie aux théories économiques du crime (Becker 1968) et à la criminologie conservatrice (Wilson 1975), dont le point commun est de mettre l'accent sur la capacité dissuasive des sanctions pénales.

L'autre explication de référence dans la littérature patronale sur le vol en interne est liée à la personnalité de l'employé contrevenant. Cette explication s'appuie sur les travaux de Hirschi (1969) et Gottfredson et Hirschi (1990). Gottfredson et Hirschi affirment que la propension au crime est fondamentalement fonction de l'incapacité des individus à contrôler leurs impulsions. Dans cette théorie, les comportements criminels sont associés à la recherche des gratifications à court-terme, comme le fait de fumer, de boire, de jouer, d'avoir des relations sexuelles sans lendemain ou de conduire trop vite (Gottfredson et Hirschi, 1990 : 91). De nombreuses recherches s'appuient sur cette théorie de la mauvaise socialisation, ou de la mauvaise personnalité, pour expliquer le vol en interne, comme celles de Terris (1979), et Gibson et Wright (2001). Merriam (1977) et Blount (2003), avec un quart de siècle d'intervalle, ont dénoncé la faible moralité des jeunes travailleurs, qui les prédisposerait à céder plus facilement à la tentation. Bernardin et Cooke $(1993: 1101)$ ont élaboré un test de personnalité à l'intention des recruteurs pour détecter les futurs employés qui seraient plus disposés que les autres à voler leur entreprise. 
A la différence de la littérature sociologique, l'économie et le management ne voient le vol en interne que comme une délinquance contre laquelle il faut lutter. La tendance de la littérature de l'économie et du management à insister sur la dimension criminelle du vol en interne contraste cependant avec le traitement pénal du vol dans les faits. Cette délinquance coûteuse n'est pas problématisée dans le débat public, ni poursuivie devant les tribunaux. Dickens et al. (1989), Ditton (1977a), Hollinger et Clark (1983), Lipman et McGraw (1988) et Tucker (1993) remarquent tous que la découverte d'un salarié voleur ne se traduit presque jamais par des poursuites pénales. Au contraire, le vol en interne se règle « en interne » : Shapland (1995:323) note que les commerçants «considèrent le vol en interne comme une affaire interne qu'ils ne voudraient pas révéler ». La sanction modale pour les vols qui ne sont pas purement véniels est le licenciement.

\section{Pistes de recherche : investissement normatif et double-bind}

La sociologie du vol en interne ne se résume pas à une opposition caricaturale entre avocats et procureurs des pratiques des salariés. Le vol en interne est aussi un cas empirique pour montrer la complexité du rapport salarial, qui ne résume pas à la confrontation d'une demande et d'une offre de travail sur un marché donné. Le vol en interne pose la question de l'investissement normatif au travail et du rapport salarial comme rapport de domination.

\subsection{Vol en interne et investissement normatif}

La question de l'investissement normatif au travail a fait l'objet de l'étude empirique la plus importante sur le vol en interne : l'enquête par autorévélation de Hollinger et Clark (1983) auprès de plus de 9000 salariés. Cette étude montre que les salariés qui volent le moins ne sont pas ceux qui ont le plus peur de la sanction, mais ceux qui ont le plus d' «engagement» (commitment) dans l'entreprise (Hollinger et Clark 1983: 415). Les salariés ne vivent pas forcément leur rapport à l'entreprise comme une lutte à mort entre travail et capital. Hollinger et Clark montrent empiriquement que les employés peuvent avoir un investissement moral dans l'entreprise.

Comme le note Etzioni (1964: 59-60), l'attrait utilitariste pour la rémunération n'est pas la seule motivation pour travailler dans les organisations : les systèmes autoritaires peuvent utiliser la coercition pour forcer la productivité des travailleurs, et de nombreuses associations reposent sur le commitment normatif de leur membres. Selon l'expérience de Lipman et McGraw (1988 : 52), le problème du vol en interne est celui du manque de loyauté et de commitment d'employés qui restent trop peu longtemps dans des entreprises qui pratiquent trop le turn-over. Ces employés n'ont donc pas le temps de construire un lien moral qui prémunirait leurs employeurs de pertes inexpliquées. Miceli et al. (1991 : 126) ont montré dans une enquête sur les dénonciations de déviances sur le lieu de travail que les dénonciateurs étaient les individus les plus loyaux et les plus investis (committed) dans l'entreprise. La dimension normative de l'investissement dans le travail montre que le rapport salarial ne se réduit pas à un échange rationnel entre 'force de travail' et 'rémunération à la productivité'. En ce sens, le vol en interne est un site empirique stratégique pour analyser cette dimension particulière du rapport salarial. 


\subsection{Double-bind et domination}

Dans une perspective radicalement différente, mais toute aussi heuristique sur la complexité du rapport salarial, Ditton (1977a) a analysé le vol en interne comme un révélateur du double-bind induit par le travail salarié. Ditton montre que l'existence des «salaires invisibles » (le chapardage, les combines et les petits avantages en nature) remonte à la naissance du capitalisme. Il se fonde sur les analyses de Marx (1867 ; 1842 ; voir aussi Henry 1987 : 142). Avant la révolution industrielle, l'économie rurale reposait sur la multiplicité des coutumes organisant la subsistance de chacun. Les paysans avaient le droit de prendre du bois qui ne leur appartenait pas si c'était pour réparer leurs outils, de faire paître leurs bêtes dans les prés communaux. Partout où les paysans et les artisans ont pu refuser le travail salarié, ils l'ont fait. Le salariat n'a pu être imposé en Angleterre qu'avec le mouvement des enclosures. La rationalisation induite par le mouvement des enclosures a consisté non seulement à priver les paysans de ce type de droits, mais en plus à criminaliser ces coutumes, pour ne laisser place qu'au salaire comme unique rétribution («les eaux glacées du calcul égoïste »). La faiblesse des salaires a contraint nombre de salariés à se livrer au chapardage ou aux combines, ce qui a fourni aux capitalistes des occasions supplémentaires de réprimer les prolétaires.

Ditton souligne la contradiction qu'il y a entre vouloir normaliser et rationaliser le salaire (en supprimant les salaires invisibles tolérés), et ne pas payer les salariés assez, de telle sorte qu'ils soient structurellement portés à voler. Les employés sont de fait placés en situation de double-bind: obligés de recourir aux salaires invisibles pour compenser un revenu insuffisant, mais menacés de licenciement s'ils sont découverts. Les à-côtés, le chapardage et les combines sont donc pour Ditton (1977a: 57) «des formes de domination cruciales pour la persistance et la croissance du capitalisme moderne, à cause de leurs solutions à ces problèmes disciplinaires qui ne sont pas solubles dans le seul argent ».

L'analyse de Ditton est lourdement influencée par le marxisme, mais ne s'y réduit pas. Ditton ne considère pas le vol en interne comme une forme de résistance dans le conflit travail-capital, mais bien comme une forme de domination subie et reproduite par les dominés eux-mêmes. Le vol en interne est une domination subie parce que l'encadrement peut instrumentaliser la légitimité de la lutte contre le vol. Le fait que les employés volent permet de justifier l'intensification de la surveillance (et donc de la surveillance de la productivité), voire fournit des prétextes pour licencier à moindre frais des salariés jugés peu productifs. Le vol en interne est une domination reproduite par les dominés euxmêmes: chaque salarié individuel dans le contexte particulier de son lieu de travail trouve tout à fait rationnel et profitable de voler son entreprise. L'intérêt de cette observation est de développer un compte-rendu purement analytique de la domination, dans lequel la seule rationalité des acteurs pris dans un rapport de pouvoir asymétrique permet de rendre compte de la reproduction et de la persistance dans le temps de ce rapport asymétrique.

\section{Conclusion}

Le vol en interne s'inscrit dans la constellation des déviances sur le lieu de travail, un objet encore négligé dans la littérature de langue française. D'un côté, on peut dire du vol en interne ce qu' Isaac Joseph a dit de la perruque : «elle 
ne joue que sur la récupération et sur des moyens de fortune, elle n'opère que des détournements de flux, elle suppose une connaissance intime du système de surveillance, une suradaptation aux techniques de contrôle. De sorte que la géographie de la liberté qu'elle permet d'explorer est dérisoire, comme sont dérisoires toutes les petites résistances qui ne s'inscrivent pas dans une logique de contradiction et qui échappent au corps à corps de la surveillance et de la docilité : la fuite, le retrait, le silence, la blague, la ruse » (cité in Anteby 2003 : 467).

De l'autre côté, James Scott, un anthropologue de la résistance des sociétés paysannes aux tentatives brutales de «modernisation », estime que «de même que des millions de polypes d'anthozoaires créent, petit à petit, un récif de corail, des milliers et des milliers de tous petits actes d'insubordination créent une barrière de récifs politiques et économiques » (cité in Morrill et al. 2003 : 401). De fait, les recherches en sciences sociales et en management sur le vol en interne sont marquées par une opposition entre une littérature qui prend le parti des salariés et fournit des justifications au vol, et une littérature qui prend le parti des patrons, pour n'en regarder que la dimension purement délinquante.

Le clivage prégnant et un peu caricatural de cette littérature ne doit pas cacher l'intérêt de l'objet. Le vol en interne est un symptôme de la complexité du rapport salarial dans l'économie moderne : il est à la fois un à-côté du revenu des travailleurs et le symbole de la dépendance qu'induit le salariat. Il est aussi bien un acte de résistance qu'un prétexte pour licencier gratuitement. Il est autant un salaire invisible qu'un vol pur et simple. Sa tolérance s'inscrit dans un marchandage asymétrique dans lequel les salariés ne sont que rarement gagnants. En même temps, l'étude des salariés qui ne volent pas suggère que le rapport salarial peut faire l'objet d'un investissement moral par les dominés, qui ne se réduit pas à une aliénation. Le vol en interne recèle une autre contradiction : il est une délinquance routinière à laquelle l'expérience quotidienne expose nécessairement, et en même temps il suscite moins le scandale que les autres formes de vol. La sociologie du travail et la criminologie gagneraient à approfondir cet objet riche des ambivalences du social. 


\section{Bibliographie}

Ackroyd S. et Thompson P. (ed.), 1999, Organizational Misbehavior, Londres : Sage

Anteby M., 2003, «La "perruque" en usine : approche d'une pratique marginale, illégale et fuyante », Sociologie du Travail, 45 (4), décembre, pp. 453-471

Astor S.D., 1972, «Who's Doing the Stealing? Twenty Steps for Preventing Theft in Business », Management Review, 61, pp. 34-35

Barkan S., 2001, Criminology. A Sociological Understanding, Second Edition, Upper Saddle River, NJ: Prentice Hall

Baumgartner M. P., 1984, « Social Control from Below », in Black D., Toward a General Theory of Social Control. Volume 1 : Fundamentals, Orlando : Academic Press, « Studies on Law and Social Control », 1984, pp. 303-345

Becker G.S., 1968, «Crime and Punishment: An Economic Approach», Journal of Political Economy, 76 (2), pp. 169-217

Beirne P. et Messerschmidt J.W., 2006, Criminology, Fourth Edition, Los Angeles, CA : Roxbury Publishing Company

Bernadin H.J., Cooke D.K., 1993, "Validity of an Honesty Test in Predicting Theft among Convenience Store Employees », Academy of Management Journal, 36 (5), October, pp. 1097-1108

Bies R.J., Tripp T.M., Kramer R.M., 1997, « At the Breaking Point: Cognitive and Social Dynamics of Revenge in Organizations », in Giacalone R. et Greenberg J. (ed.), Antisocial Behaviors in Organizations, Thousands Oaks, CA : Sage, pp. 37-67

Black D. (dir.), 1984a, Toward a General Theory of Social Control. Volume 1: Fundamentals, Orlando: Academic Press, «Studies on Law and Social Control »

Black D. (dir.), 1984b, Toward a General Theory of Social Control. Volume 2 : Selected Problems, Orlando: Academic Press, Inc, «Studies in Law and Social Control»

Black D., 1983, «Crime as Social Control», American Sociological Review, 48 (1), February, pp. 34-45

Blount E.C., 2003, Occupational Crime : Deterrence, Investigation, and Reporting in Compliance with Federal Guidelines, Boca Raton : CRC Press

Bozon M. et Lemel Y., 1989, «Les petits profits du travail salarié. Moments, produits et plaisirs dérobés », Revue française de sociologie, XXX, pp. 101-127

Dickens W.T., Katz L.F., Lang K., Summers L.H., 1989, «Employee Crime and the Monitoring Puzzle », Journal of Labor Economics, 7 (3), July 1989, pp. 331-347

Ditton J., 1977a, « Perks, Pilferage, and the Fiddle : The Historical Structure of Invisible Wage », Theory and Society, 4 (1), Spring, pp. 39-71

Ditton J., 1977b, Part-Time Crime. An Ethnography of Fiddling and Pilferage, Londres : MacMillan

Etzioni A., 1964, Modern Organizations, Englewood Cliffs, N.J.: Prentice-Hall, «Foundations of modern sociology series » 
Giacalone R. et Greenberg J. (ed.), 1997, Antisocial Behaviors in Organizations, Thousands Oaks, CA : Sage

Gibson C., Wright J., 2001, «Low Self-Control and Coworker Delinquency : A Research Note », Journal of Criminal Justice, 29 (5), pp. 483-501

Gottfredson, M.R., Hirschi T., 1990, A General Theory of Crime, Stanford : Stanford University Press

Greenberg D., 1981, «Praxis and Marxian Criminology », in Greenberg David (dir.), Crime and Capitalism. Readings in Marxist Criminology, Philadelphia: Temple University Press, édition mise à jour et augmentée : 1993

Greenberg J., 1990, «Employee Theft as a Reaction to Underpayment Inequality: The Hidden Costs of Pay Cuts », Journal of Applied Psychology, 75 (5), pp. 561-568

Henry S., 1987, «The Political Economies of Informal Economies », Annals of the American Academy of Political and Social Sciences, $\mathrm{n}^{\circ} 493$, September 1987, pp. 137-153

Hirschi T., 1969, Causes of Delinquency, Berkeley, Los Angeles: University of California Press

Hodson R., 2004, «A Meta-Analysis of Workplace Ethnographies. Race, Gender, and Employee Attitudes and Behaviors », Journal of Contemporary Ethnography, 33 (1), February, pp. 4-38

Hollinger R.C., Clark J.P., 1983, «Deterrence in the Workplace : Perceived Certainty, Perceived Severity, and Employee Theft», Social Forces, 62 (2), December, pp. 398-418

Horning, D., 1970, «Blue Collar Theft: Conceptions of Property, Attitudes Toward Pilfering, and Work Group Norms in a Modern Industrial Plant », in E. O. Smigel, H. L. Ross (dir.), Crimes Against Bureaucracy, New York : Van Nostrand Reinhold, pp. 44-64

Joseph I., 1980, La perruque, Urbi III, Mars, pp. 115-119

Katz J., 1988, Seductions of crime. The Moral and Sensual Attractions of Doing Evil, New York : Basic Books

Kwok C.-K., Au W.T., Ho J.M.C., 2005, « Normative Controls and Self-Reported Counterproductive Behaviors in the Workplace in China », Applied Psychology : An International Review, 54 (4), 2005, pp. 456-475

Lipman M., McGraw W.R., 1988, «Employee Theft : A \$40 Billion Industry », Annals of the American Academy of Political and Social Sciences, vol. 498, July, pp. 51-59

Mangione T.W., Quinn R.P., 1975, «Job Satisfaction, Counter-Productive Behavior and Drug Use at Work », Journal of Applied Psychology, vol. 60, pp. 114-16

Mars G., 1982, Cheats at Work. An Anthropology of Workplace Crime, Londres : George Allen and Unwin

Marx K., 1842, «Débats sur la loi relative aux vols de bois », pp. 139-189, in Lascoumes P., Zander H., Marx : du «vol de bois » à la critique du droit. Naissance d'une méthode. Édition critique des "Débats sur la loi relative au vols de bois » et «Justification du correspondant de la Moselle », Paris : PUF, 1984, «Philosophie d'aujourd'hui » 
Marx K., 1867, Le capital. Livre 1, Paris : Flammarion, « GF », 1969

Merriam D., 1977, «Employee Theft », Criminal Justice Abstracts, 9, pp. 380-386

Merton R.K., 1938, «Social Structure and Anomie», in Social Theory and Social Structure, Glencoe : The Free Press, 1957, $2^{\text {nd }}$ éd.

Miceli M.P., Near J.P., Swank C.C., 1991, «Who blows the Whistle and Why?», Industrial and Labor Relations Review, 45 (1), October, pp. 113-130

Morrill C., Zald M.N., Rao H., 2003, «Covert Political Conflict in Organizations : Challenges from Below », Annual Review of Sociology, vol. 29, pp. 391415

Ocqueteau F., Pottier M.-L., 1995, Vigilance et sécurité dans les grandes surfaces, Paris : L'Harmattan

Payne B.K. et Gainey R.R., 2004, «Ancillary Consequences of Employee Theft», Journal of Criminal Justice, vol. 32, pp. 63-73

Robinson S.L., Bennett R.J., 1995, «A Typology of Deviant Workplace Behaviors : A Multidimensional Scaling Study », Academy of Management Journal, 38 (2), April, pp. 555-572

Roscigno V.J. et Hodson R., 2004, «The Organizational and Social Fouandations of Worker Resistance », American Sociological Review, 69 (1), February, pp. 14-39

Sellin T., 1938, Culture Conflicts and Crime, New York: Social Science Research Council, trad. fr., Conflits de culture et criminalité, Paris: Pedone, «Publications du centre de recherches de politique criminelle », 1984

Shapiro S.P., 2005, «Agency Theory », Annual Review of Sociology, vol. 31, pp. 263284

Shapland J., 1995, «Preventing Retail-Sector Crimes », Crime and Justice, vol. 19, pp. 263-342

Siegel L.J., 2003, Criminology, Eighth Edition, Belmont, CA: Wadsworth

Sieh E.W., 1987, «Garment Workers : Perceptions of Inequity and Employee Theft », British Journal of Criminology, 27 (2), pp. 174-190

Sieh E.W., 1993, «Employee Theft : An Examination of Gerald Mars and an Explanation Based on Equity Theory », Advances in Criminological Theory, vol. 4, pp. 95-111

Spector P.E., 1997, "The Role of Frustration in Antisocial Behavior at Work », in Giacalone R. et Greenberg J. (ed.), Antisocial Behaviors in Organizations, Thousands Oaks, CA : Sage, pp. 1-17

Sutherland D., 1924, Criminology, 11ème édition: (avec D. R. Cressey et D. F. Luckenbill), Principles of Criminology, New York : General Hall, 1992

Sykes G.M. et Matza D., 1957, «Techniques of Neutralization: A Theory of Delinquency », American Sociological Review, 22 (6), December, pp. 664670

Terris W., 1979, «Attitudinal Correlates of Employee Integrity », Journal of Security Administration, vol. 2, pp. 30-39

Tucker J., 1989, «Employee Theft as Social Control», Deviant Behavior, 10, pp. 319334 
Tucker J., 1993, « Everyday Forms of Employee Resistance », Sociological Forum, 8 (1), March, pp. 21-45

Tucker J., 1999, «Worker Deviance as Social Control», Research in the Sociology of Work, Vol. 8, pp. 1-16

Wilson J.Q., 1975, Thinking About Crime, New York: Vintage Books, $2^{\text {nde }}$ éd., 1983 